PROCEEDINGS OF THE

AMERICAN MATHEMATICAL SOCIETY

Volume 130, Number 10, Pages 3075-3084

S 0002-9939(02)06404-3

Article electronically published on March 14, 2002

\title{
APPROXIMATION OF CONVEX BODIES BY AXIALLY SYMMETRIC BODIES
}

\author{
MAREK LASSAK
}

(Communicated by Wolfgang Ziller)

\begin{abstract}
Let $C$ be an arbitrary planar convex body. We prove that $C$ contains an axially symmetric convex body of area at least $\frac{2}{3}|C|$. Also approximation by some specific axially symmetric bodies is considered. In particular, we can inscribe a rhombus of area at least $\frac{1}{2}|C|$ in $C$, and we can circumscribe a homothetic rhombus of area at most $2|C|$ about $C$. The homothety ratio is at most 2 . Those factors $\frac{1}{2}$ and 2 , as well as the ratio 2 , cannot be improved.
\end{abstract}

In the survey article about measures of symmetry of convex bodies, Grünbaum suggests to consider measures of axiality of convex bodies and other measures of this kind (see 12, p. 263). The general problem of finding a large $k$-symmetric convex body in a convex body of Euclidean $d$-space $E^{d}$ is considered by Chakerian and Stein 3]. A few measures of axiality are proposed by deValcourt [5].

Our aim is to approximate convex bodies of Euclidean plane $E^{2}$ by axially symmetric bodies. We are interested in three such kinds of approximation. For a given convex body $C \subset E^{2}$ we are looking for an axially symmetric convex body $A$ of possibly large area contained in $C$, for an axially symmetric convex body $B$ of possibly small area containing $C$, and for an axially symmetric convex body $D$ such that $D \subset C \subset h(D)$, where $h$ is a homothety with possibly small positive homothety ratio. We are also looking for some specific axially symmetric bodies like rhombi, isosceles triangles and some axially symmetric hexagons which well approximate $C$.

The area of a convex body $C \subset E^{2}$ is denoted by $|C|$ and the length of the segment $a b$ by $|a b|$. Nohl [18] proves that every centrally symmetric convex body $C \subset E^{2}$ contains an axially symmetric convex body of area at least $2(\sqrt{2}-1)|C|$ and that the coefficient $2(\sqrt{2}-1)$ cannot be enlarged. Krakowski [15] shows that every convex body $C \subset E^{2}$ contains an axially symmetric convex body of area at least $\frac{5}{8}|C|$. We improve this coefficient up to $\frac{2}{3}$. We also show that $C$ is contained in an axially symmetric convex body of area at most $\frac{31}{16}|C|$.

Approximation of any convex body $C \subset E^{2}$ by specific axially symmetric bodies is considered by several authors. First of all, we have the two-dimensional case of the classic theorem of John [14] which says that for every convex body $C$ there exists an ellipse contained in $C$ such that its two times larger homothetic copy contains $C$.

Received by the editors March 1, 2000 and, in revised form, May 1, 2001.

1991 Mathematics Subject Classification. Primary 52A10, 52A27.

Key words and phrases. Convex body, axial symmetry, rhombus, isosceles triangle, area, approximation.

This research was supported by Deutsche Forschungsgemeinschaft. 
There exists a rectangle $R$ inscribed in $C$ such that its homothetic copy of positive ratio at most 2 is circumscribed about $C$ (see [19], [17] and [20]). In Theorem 1 we give an analogous theorem about approximation by a pair of homothetic rhombi. The above-mentioned theorems give corollaries about the existence of ellipses, rectangles and rhombi of area at least $\frac{1}{2}|C|$ contained in $C$, and of area at most $2|C|$ containing $C$. Similar questions about isosceles triangles, axially symmetric octagons, and kites contained in $C$ or containing $C$ are considered by deValcourt [6, 7].

A regular convex body in the plane is a convex body whose each boundary point lies in only one supporting line, and such that every supporting line meets this body only at one point (see [8], p. 31).

\section{Approximation BY RHOMBI}

Theorem 1. Let $C \subset E^{2}$ be a convex body. There exists a rhombus $R$ inscribed in $C$ such that a homothetic copy $R^{\prime}$ of $R$ is circumscribed about $C$. The positive ratio of homothety is at most 2.

Proof. First we consider a regular convex body $C$. For every direction $\theta$ we can inscribe exactly one rhombus $R(\theta)$ in $C$ with a diagonal of direction $\theta$. This follows from [4, 9] and [21.

Denote by $a(\theta), b(\theta), c(\theta)$ and $d(\theta)$ the consecutive vertices of $R(\theta)$; assume that the vector $a(\theta) b(\theta)$ is parallel to $\theta$ and oriented as $\theta$.

As we rotate $\theta$, the vertices of $R(\theta)$ change continuously. This easily follows from the Bolzano-Weierstrass theorem (comp. [4, where the continuity of the position of the angles of the rhombus $R(\theta)$ is shown).

For every $R(\theta)$ we construct the circumscribed parallelogram $R^{\prime}(\theta)$ of $C$ with sides parallel to the sides of $R(\theta)$. It is clear that $R^{\prime}(\theta)$ exists and is unique for every $\theta$. Denote by $a^{\prime}(\theta), b^{\prime}(\theta), c^{\prime}(\theta), d^{\prime}(\theta)$ the corresponding vertices of $R^{\prime}(\theta)$ (we mean that the vectors $a(\theta) b(\theta)$ and $a^{\prime}(\theta) b^{\prime}(\theta)$ are parallel and have a common orientation). Consider four strips, each determined by a pair of parallel segments contained in the bounding lines of the strip: the strip $S(\theta)$ determined by the segments $a(\theta) b(\theta)$ and $c(\theta) d(\theta)$, the strip $S^{\prime}(\theta)$ determined by the segments $a^{\prime}(\theta) b^{\prime}(\theta)$ and $c^{\prime}(\theta) d^{\prime}(\theta)$, the strip $T(\theta)$ determined by the segments $b(\theta) c(\theta)$ and $d(\theta) a(\theta)$, the strip $T^{\prime}(\theta)$ determined by the segments $b^{\prime}(\theta) c^{\prime}(\theta)$ and $d^{\prime}(\theta) a^{\prime}(\theta)$. Denote by $f(\theta)$ the ratio of the width of $S^{\prime}(\theta)$ to the width of $S(\theta)$. Let $g(\theta)$ mean the ratio of the width of $T^{\prime}(\theta)$ to the width of $T(\theta)$. From the continuity of the position of the vertices of $R(\theta)$ we see that $f(\theta)$ and $g(\theta)$ are continuous functions. Since $f\left(\theta_{1}\right)=g\left(\theta_{2}\right)$ for perpendicular directions $\theta_{1}$ and $\theta_{2}$, we conclude that there exists a direction $\theta_{0}$ such that $f\left(\theta_{0}\right)=g\left(\theta_{0}\right)$. Thus $R^{\prime}\left(\theta_{0}\right)$ is a homothetic copy of $R\left(\theta_{0}\right)$. From the Lemma in [17, where $s_{1}=s_{2}$, it follows that the positive ratio of homothety is at most 2 . The statement of Theorem 1 is shown in the case of a regular convex body $C$.

An arbitrary convex body $C \subset E^{2}$ can be presented as a limit of a sequence of regular convex bodies $C_{1}, C_{2}, \ldots$ (see [8]). As is shown above, for every $C_{i}$ there is a pair of homothetic inscribed and circumscribed homothetic rhombi $R_{i}$ and $R_{i}^{\prime}$. By four consecutive choices (each for one vertex), from the sequence $R_{1}, R_{2}, \ldots$ we can select a subsequence which is convergent to a rhombus $R$ inscribed in $C$. Since every $R_{i}^{\prime}$ is a homothetic copy of $R_{i}$ with a positive ratio at most 2 , from the selected subsequence we can select a subsequence such that the corresponding subsequence of circumscribed rhombi is convergent to a rhombus $R^{\prime}$. Of course, $R^{\prime}$ 
is a homothetic copy of $R$ with a positive ratio at most 2 . Thus we have obtained the promised pair $R, R^{\prime}$ of homothetic rhombi.

The ratio 2 in Theorem 1 cannot be lessened because of the example of an arbitrary triangle as $C$. Also it cannot be lessened for centrally symmetric convex bodies. It follows from the example of any rectangle with the ratio of lengths of the perpendicular sides at least 2 .

Analogously as in Part 6 of the proof of the Theorem in [17], we show that the inscribed rhombus $R$ in our Theorem 1 has area at least $\frac{1}{2}|C|$, and that the circumscribed rhombus $R^{\prime}$ has area at most $2|C|$. Thus we obtain the following Corollary 1, which answers the question of deValcourt [7] about a rhombus of possibly large area contained in $C$ and about a rhombus of possibly small area containing $C$.

Corollary 1. Let $C$ be a planar convex body. We can inscribe a rhombus of area at least $\frac{1}{2}|C|$ in $C$, and we can circumscribe a rhombus of area at most $2|C|$ about $C$.

\section{Approximation By isosceles tRiangles}

Every convex body $C \subset E^{2}$ is contained in a triangle of area at most $2|C|$ (Gross [11]). We conjecture that $C$ is contained in an isosceles triangle of area at most $2|C|$. The second sentence of the following corollary gives only the factor $\frac{49}{16}$.

Corollary 2. Let $C \subset E^{2}$ be a convex body. There exists an isosceles triangle $T \subset C$ such that its homothetic copy $T^{\prime}$ of ratio at most $\frac{7}{2}$ contains $C$. We have $\left|T^{\prime}\right| \leq \frac{49}{16}|C|$.

Proof. Consider the inscribed rhombus $R=a b c d$ and the homothetic circumscribed rhombus $R^{\prime}=a^{\prime} b^{\prime} c^{\prime} d^{\prime}$ from Theorem 1. Denote by $\rho$ the positive ratio of this homothety. When we prolong the sides of the rhombus $R$, they intersect the sides of the rhombus $R^{\prime}$. We obtain four parallelograms at the vertices of $R^{\prime}$ (see Figure 1).

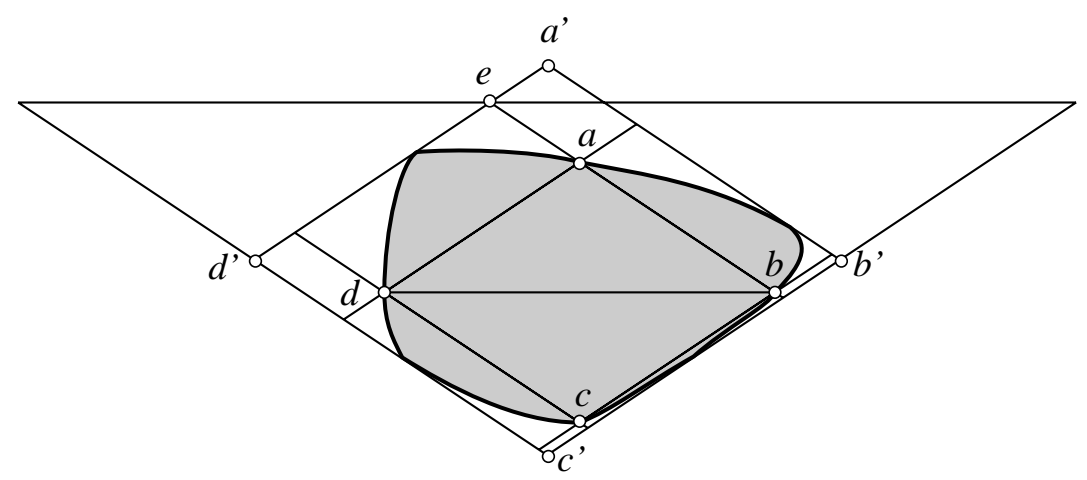

FigURE 1 .

One of the parallelograms contains translates of the three other parallelograms. We do not lose the generality of the considerations assuming that this largest parallelogram $K$ is at the vertex $a^{\prime}$. Of course, the triangle $T=b c d$ is isosceles. Now we describe the homothetic copy $T^{\prime}$ promised in the formulation of Corollary 2. Two sides of $T^{\prime}$ contain the segments $b^{\prime} c^{\prime}$ and $c^{\prime} d^{\prime}$. The third side of $T^{\prime}$ is parallel to the 
segment $b d$ and passes through a vertex $e$ of $K$ in such a way that $T^{\prime}$ contains all vertices of $K$ different from $a^{\prime}$. Since $a$ is on the boundary of $C$, from our construction and from the convexity of $C$ we conclude that the third side of $T^{\prime}$ is disjoint with the interior of $C$. So we have $T \subset C \subset T^{\prime}$. Of course, $T^{\prime}$ is a homothetic copy of $T$. Let us estimate the ratio $\delta$ of this homothety and the area of $\left|T^{\prime}\right|$. Put $\kappa=\left|e a^{\prime}\right| /|d a|$. The choice of $K$ implies that $\kappa \geq \frac{1}{2}(\rho-1)$. Observe that the side of $T^{\prime}$ parallel to $d^{\prime} a^{\prime}$ has length $2\left|d^{\prime} a^{\prime}\right|-\left|e a^{\prime}\right|$. Thus we have $\delta=\left(2\left|d^{\prime} a^{\prime}\right|-\left|e a^{\prime}\right|\right) /|d a|=$ $2\left|d^{\prime} a^{\prime}\right| /|d a|-\left|e a^{\prime}\right| /|d a|=2 \rho-\kappa \leq 2 \rho-\frac{1}{2}(\rho-1)=\frac{3}{2} \rho+\frac{1}{2} \leq \frac{7}{2}$. This inequality follows from $1 \leq \rho \leq 2$. Since $R$ is inscribed in $C$ and since $C$ touches the sides of $R^{\prime}$, we get $|\bar{C}| \geq|R|+2 \cdot \frac{1}{2}(\rho-1)|R|=\rho|R|$. Thus $\left|T^{\prime}\right| /|C|=\delta^{2}|T| /(\rho|R|)=$ $\frac{1}{2} \delta^{2}|R| /(\rho|R|)=\frac{1}{2}(2 \rho-\kappa)^{2} \rho^{-1} \leq \frac{1}{2}\left(2 \rho-\frac{1}{2}(\rho-1)\right)^{2} \rho^{-1}=\frac{1}{8}\left(9 \rho+6+\rho^{-1}\right) \leq \frac{49}{16}$. The last inequality results from $1 \leq \rho \leq 2$.

How much can the ratio in Corollary 2 be lessened? Surely not below $1+\frac{1}{2} \sqrt{5}$ because of the example of the regular pentagon as $C$; see [10] and [16] where analogous approximation by a pair of triangles (not necessarily isosceles) is considered. We omit here a construction of another pentagon such that, for each such pair of homothetic isosceles triangles, the ratio is larger than $1+\frac{1}{2} \sqrt{5}$.

DeValcourt [7] presents two "theorems" which say that every convex body $C \subset$ $E^{2}$ contains an isosceles triangle of area at least $\frac{3}{8}|C|$ and that it is contained in an isosceles triangle of area at most $2|C|$. He gives a mistaken proof of the first "theorem" and says that the proof of the second theorem is analogous. More precisely, he considers a triangle $T_{\varphi}$ of the maximum area inscribed in a strictly convex body $C$ such that a side $a_{\varphi}^{\prime} a_{\varphi}^{\prime \prime}$ of $T_{\varphi}$ has a given direction $\varphi$. The proof is based on the false claim about the continuity of the function $f(\varphi)=a^{\prime} / a^{\prime \prime}$, where $a^{\prime}$ and $a^{\prime \prime}$ are the distances of $a_{\varphi}^{\prime}$ and $a_{\varphi}^{\prime \prime}$ from the orthogonal projection of the remaining vertex of $T_{\varphi}$ on the straight line containing $a_{\varphi}^{\prime}$ and $a_{\varphi}^{\prime \prime}$. Here is an example of a strictly convex body $D$ for which $f(\varphi)$ is not continuous. Let $\epsilon>0$ be a small number (for instance, 0.01). We define $D$ as the convex hull of the unit circle $U$ centered at $(0,0)$ and of five arcs $A_{0}, A_{1}, A_{2}, A_{3}, A_{4}$ of circles. By $A_{0}$ we mean the small arc of the circle of radius $1+\epsilon$, centered at $(0,0)$, between points $p_{1}=(\epsilon, \sqrt{1+2 \epsilon})$ and $p_{2}=(0,1+\epsilon)$. Moreover, put $p_{3}=p_{4}=(\epsilon,-1-\epsilon)$. The arcs $A_{i}$, where $i \in\{1,2,3,4\}$, are arcs of four circles of a large radius (say, $1 / \epsilon$ ) such that $U$ is tangent to them from within: $A_{i}$ connects $p_{i}$ with the tangent point on $U$ in the $i$-th quarter of the coordinate system. For the direction $\varphi=0$ of the axis $O x$ we have two triangles $T_{\varphi}$ with the maximum possible area in $D$; the first has a vertex at $p_{2}$ and the second at $p_{3}$. The ratios $a^{\prime} / a^{\prime \prime}$ for those two triangles are different. Even if we choose one of them, the trouble remains because the ratio $a^{\prime} / a^{\prime \prime}$ does not change continuously at $\varphi=0$. Just the one-sided limits of this ratio at $\varphi=0$ are different. Of course, the proof of deValcourt, as well as the omitted proof, is valid provided $C$ is centrally symmetric.

By the way, the proof is also incorrect if by $T_{\varphi}$ we mean the triangle $a b c$ inscribed in $C$ of the maximum possible area such that the vector $a b$ is of the direction $\varphi$ and such that $a, b, c$ are in the counterclockwise order on the boundary of $C$. When such a triangle $T_{\varphi}$ becomes isosceles for a direction $\varphi_{0}$, the applied in [7] result of Hodges [13] does not guarantee that $\left|T_{\varphi}\right| \geq \frac{3}{8}|C|$. This proof only claims that $|a b c| \geq \frac{3}{8}|C|$ or that $\left|a^{\prime} b^{\prime} c^{\prime}\right| \geq \frac{3}{8}|C|$, where $a^{\prime} b^{\prime} c^{\prime}$ is an analogous triangle with vertices in the clockwise order. 
DeValcourt [7] conjectures that every convex body $C \subset E^{2}$ contains an isosceles triangle of area at least $\frac{3 \sqrt{3}}{4 \pi}|C|$ (which is true when we do not require that the triangle is isosceles, as proved by Gross [1]). The following example disproves this conjecture. Let $F$ be the ellipse $\frac{1}{4} x^{2}+y^{2} \leq 1$. It is an affine image of the unit disk $M$. The corresponding images of the regular triangles inscribed in $M$ are the only triangles of area $\frac{3 \sqrt{3}}{4 \pi}|F|$ in $F$. Exactly four of them are isosceles; they are the images of the regular triangles inscribed in $M$ with a vertical or a horizontal side. None of them has a vertex at $\left(\sqrt{2}, \frac{1}{2} \sqrt{2}\right)$. As we explain below, for a convex body $G$ which is obtained from $F$ by a sufficiently slight "enlargement" at the point $\left(\sqrt{2}, \frac{1}{2} \sqrt{2}\right)$, those four isosceles triangles are still the only isosceles triangles of maximum area in $G$. This area is smaller than $\frac{3 \sqrt{3}}{4 \pi}|G|$, which disproves the conjecture. Now we show that such an above-promised body $G$ exists. Put $u_{n}=\left(\frac{n+1}{n} \cdot \sqrt{2}, \frac{n+1}{n} \cdot \frac{1}{2} \sqrt{2}\right)$ and denote by $G_{n}$ the convex hull of $F \cup\left\{u_{n}\right\}$, where $n=1,2, \ldots$ We claim that there is an index $m$ such that $G_{m}$ can be taken in the part of $G$. Assume the opposite. Then for every $n$ there exists an isosceles triangle $T_{n} \subset G_{n}$ of area at least $\frac{3 \sqrt{3}}{4 \pi}|F|$ and with a vertex out of $F$. From the sequence $T_{1}, T_{2}, \ldots$ we can select a subsequence which converges to an isosceles triangle $T$. Of course, $T \subset F$, the point $\left(\sqrt{2}, \frac{1}{2} \sqrt{2}\right)$ is a vertex of $T$, and the area of $T$ is at least $\frac{3 \sqrt{3}}{4 \pi}|F|$. We obtain a contradiction with the earlier observation that such an isosceles triangle does not exist.

Similar to Corollary 2, from the result about the approximation of a convex body by a pair of rectangles $([19],[17$, [20]), we obtain the following corollary. Every convex body $C \subset E^{2}$ contains a rectangular triangle $T \subset C$ such that a homothetic copy $T^{\prime}$ of ratio $\frac{7}{2}$ contains $C$. How much can one lessen this ratio? Of course, not below $\sqrt{2}+1$ as the example of a disk shows. But even this is not the infimum as it follows from a construction analogous to the construction of the set $G$. Also the questions appear about a rectangular triangle of possibly large area in $C$ and about a rectangular triangle of possibly small area containing $C$ (a rectangular triangle of area at most $\frac{49}{16}|C|$ containing $C$ can be constructed similarly as in the proof of Corollary 2).

\section{Approximation By ARBITRARY AXIALLY SYMMETRIC BODIES}

Remember that by an affine regular hexagon we mean any non-degenerate hexagon which is an affine image of the regular hexagon. In other words, a nondegenerate hexagon abcdef is affine regular if it is centrally symmetric, and if the side $a b$ is parallel to the chord $f c$ and is exactly two times shorter. We call a nondegenerated hexagon abcdef axially regular if the straight line through $f$ and $c$ is an axis of symmetry of this hexagon, and if the side $a b$ is parallel to the chord $f c$ and is exactly two times shorter. Here is a lemma analogous to the well known result of Besicovitch [1] that an affine regular hexagon can be inscribed in an arbitrary convex body $C \subset E^{2}$.

Lemma. In every convex body $C \subset E^{2}$ we can inscribe an axially regular hexagon.

Proof. Assume that $C$ is a regular convex body; at the end of the proof we will consider an arbitrary convex body $C \subset E^{2}$.

For any given direction $\theta$ there are three chords $a b, f c, e d$ in $C$ of this direction so that $|a b|=|e d|, f c$ is equidistant from the straight lines containing $a b$ and $e d$, and $|f c|=2|a b|$ (see [1]). Since $C$ is a regular body, those three chords are unique. 
The chords $a d$, be and $f c$ meet in a point $o$. The chords $a d$ and be are bisected at $o$. For arbitrary $\theta$ consider the angles $\alpha=\angle f o a, \beta=\angle a o b$ and $\gamma=b o c$. Let $H(\theta)$ denote the hexagon abcdef determined by $\theta$. Of course, for any direction $\theta$ there is a unique $H(\theta)$.

Besicovitch 1] rotates $\theta$ and applies continuity arguments in order to show that there is a direction $\theta_{1}$ for which the position $o_{1}$ of $o$ is the center of the corresponding chord $f_{1} c_{1}$. Thus $H\left(\theta_{1}\right)=a_{1} b_{1} c_{1} d_{1} e_{1} f_{1}$ is an affine regular hexagon. Denote by $\theta_{2}$ the direction of the chord $a_{1} d_{1}$ and by $\theta_{3}$ the direction of the chord $b_{1} e_{1}$.

Put $H\left(\theta_{i}\right)=a_{i} b_{i} c_{i} d_{i} e_{i} f_{i}$ and $\alpha_{i}=\angle f_{i} o a_{i}, \beta_{i}=\angle a_{i} o b_{i}$ and $\gamma_{i}=b_{i} o c_{i}$ for $i \in$ $\{1,2,3\}$. From the uniqueness of $H(\theta)$ and since $H\left(\theta_{1}\right)$ is an affine regular hexagon, we see that $H\left(\theta_{1}\right)=H\left(\theta_{2}\right)=H\left(\theta_{3}\right)$. Thus there is an index $i \in\{1,2,3\}$ such that $\alpha_{i} \leq \min \left\{\beta_{i}, \gamma_{i}\right\}$. Let for instance $\alpha_{1} \leq \min \left\{\beta_{1}, \gamma_{1}\right\}$. Further consideration is similar in the remaining cases.

When rotating $\theta$ from $\theta_{1}$ to $\theta_{2}$, all the six vertices of $H(\theta)$ and the point $o$ change the positions continuously. Also the angles $\alpha, \beta, \gamma$ change continuously: $\alpha$ from $\alpha_{1}$ to $\alpha_{2}=\beta_{1}, \beta$ from $\beta_{1}$ to $\beta_{2}=\gamma_{1}$, and $\gamma$ from $\gamma_{1}$ to $\gamma_{2}=\alpha_{1}$. Thus there is an angle $\theta^{*}$ between $\theta_{1}$ and $\theta_{2}$ for which the corresponding angles $\alpha^{*}$ and $\gamma^{*}$ are equal. Of course, $H\left(\theta^{*}\right)$ is an axially regular hexagon inscribed in $C$.

The above solution for a regular convex body leads to a solution for an arbitrary convex body $C \subset E^{2}$. Just $C$ is a limit of a sequence of regular convex bodies (see [8]). From the corresponding sequence of axially regular hexagons inscribed in those regular bodies we can select a subsequence convergent to an axially regular hexagon inscribed in $C$.

Below we improve the result of Krakowski [15] that every convex body $C \subset E^{2}$ contains an axially symmetric body of area at least $\frac{5}{8}|C|$. We show that $C$ contains an axially regular hexagon of area at least $\frac{2}{3}|C|$.

Theorem 2. Every convex body $C \subset E^{2}$ contains an axially symmetric convex body of area at least $\frac{2}{3}|C|$.

Proof. According to the Lemma, we can inscribe an axially regular hexagon $H=$ abcdef in $C$. Without loss of generality we may assume that $|f o| \geq|o c|$.

Consider the triangle $p q r$ such that $f a \subset p q, b c \subset q r$ and $d e \subset r p$, and also the triangle $p^{\prime} q^{\prime} r^{\prime}$, where $p^{\prime}, q^{\prime}, r^{\prime}$ are points symmetric to the points $p, q, r$ with respect to the straight line through $f$ and $c$. Since $H$ is inscribed in $C$, from the convexity of $C$ it follows that $C$ is contained in the starshaped set $S=p q r \cup p^{\prime} q^{\prime} r^{\prime}$ (see Figure 2).

We provide supporting lines of $C$ at points $a, c, e, f$ and we consider the halfplanes bounded by them and containing $C$. Let $Q$ denote the intersection of those four half-planes and of $S$. The supporting lines at $f$ and at $c$ cut off from $S$ four triangles of total area $\frac{1}{3}|H|$. Denote by $W$ the starshaped set which remains from $S$ after cutting off those four triangles. Consider the two triangles $f s a$ and $f e t$, where $s$ and $t$ are the intersections of the supporting line through $f$ with the segments $p^{\prime} a$ and pe, respectively. We do not make our considerations narrower assuming that $\angle o f s \leq 90^{\circ}$ and that $\angle t f o \geq 90^{\circ}$.

The supporting line of $C$ at $a$ cuts off two triangles from $W$. Since $|f a|=$ $|a q|$ and $|s a| \leq|a b|$, the total area of those two triangles is minimized when the supporting line contains $f a$; this is when we cut off the triangle $f s a$. Analogously, the supporting line of $C$ through $e$ cuts off two triangles from $W$. Since $|f e|=\left|e q^{\prime}\right|$, 


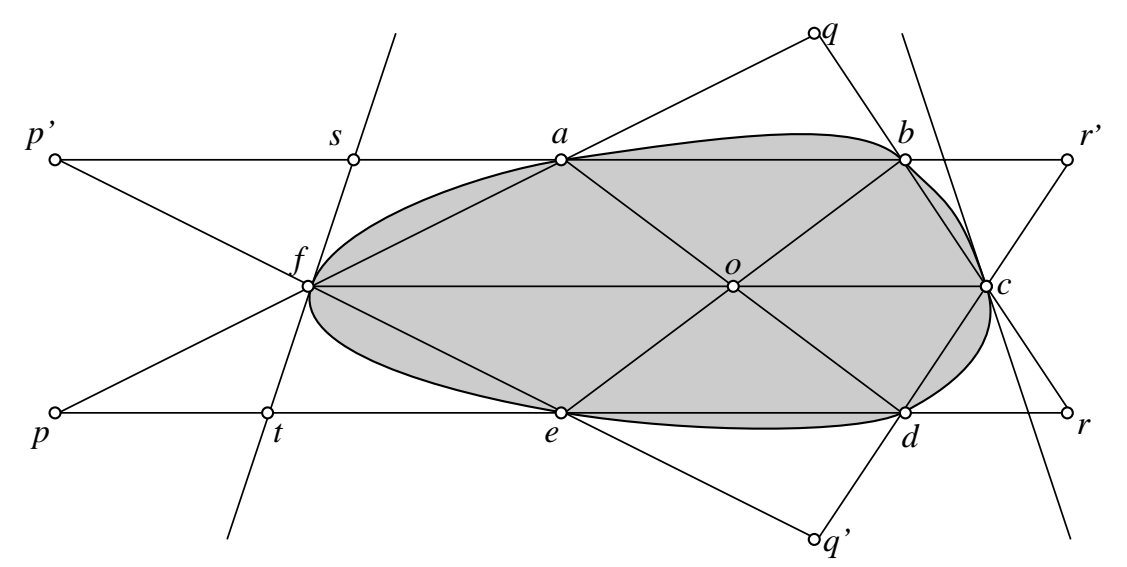

FigURE 2.

the total area of those two triangles is minimized when we cut off the triangle fet or if we cut off the triangle $e d q^{\prime}$. The first case is when $|t e| \leq|e d|$, and the second case is when $|t e| \geq|e d|$. In each of the two worst cases, the sum of the areas of all the pieces cut off from $S$ by the four considered supporting lines is at least $\frac{1}{2}|H|$. The reason is that the area of the pieces which are cut off is at least $|p f e|+\left|p^{\prime} a f\right|+\frac{1}{2}\left(|c d r|+\left|b c r^{\prime}\right|\right)=(|p f e|+|d c r|)+\left|p^{\prime} a f\right| \geq \frac{1}{3}|H|+\frac{1}{6}|H|=\frac{1}{2}|H|$ in the first case, and at least $\frac{1}{2}\left(|p f e|+\left|p^{\prime} a f\right|+|c d r|+\left|b c r^{\prime}\right|\right)+\left|e d q^{\prime}\right|=\frac{1}{3}|H|+\frac{1}{6}|H|=\frac{1}{2}|H|$ in the second case. Thus from $|S|=2|H|$ we conclude that $|Q| \leq \frac{3}{2}|H|$. By $C \subset Q$, we have $|C| \leq \frac{3}{2}|H|$. Consequently, $|H| \geq \frac{2}{3}|C|$.

It is likely that a better estimate than $\frac{2}{3}|C|$ holds true. But the examples of some parallelograms (see [18]) and triangles (see [2]) show that an estimate over $(2 \sqrt{2}-2)|C|$ is impossible.

Theorem 2 implies the existence of an axially symmetric set (usually non-convex) containing $C$ whose area is at most $\frac{4}{3}|C|$. This set is the union of the convex body $C$ and of its image in the straight line containing the segment $f c$ (see the proof of Theorem 2). What is more, the following three conditions are equivalent: (1) $C$ contains an axially symmetric convex body of area $\lambda|C|,(2) C$ is contained in an axially symmetric set (not necessarily convex) of area $(2-\lambda)|C|,(3) C$ can be dissected into two parts by a straight line $L$ such that after folding one of the two obtained parts of $C$ along $L$, the area of the union of the folded part and of the other part of $C$ is $\left(1-\frac{1}{2} \lambda\right)|C|$. We leave the short proof as an exercise for the reader. This last condition leads to the question of how large may a piece of $C$ be folded along a straight line into $C$. An estimate is given in the following theorem.

Theorem 3. For every convex body $C \subset E^{2}$ there is a straight line $L$ cutting off remains in $C$ after folding it along $L$.

Proof. We will use the notation introduced in the proof of Theorem 2. Provide this supporting line $M$ of $C$ parallel to the segment ae which passes through pe. Denote by $u$ a point of support and by $w$ the intersection of $M$ with pe (see Figure 3 ).

Since the hexagon abcde $f$ is axially regular and since $|f o| \geq|o c|$, we have $\frac{1}{2}|f c| \leq$ $|f o| \leq \frac{3}{4}|f c|$ and $|f c|=2|e d|$. From the triangle $a d p$ we get $|f o|=\frac{1}{2}|p d|$ (see 


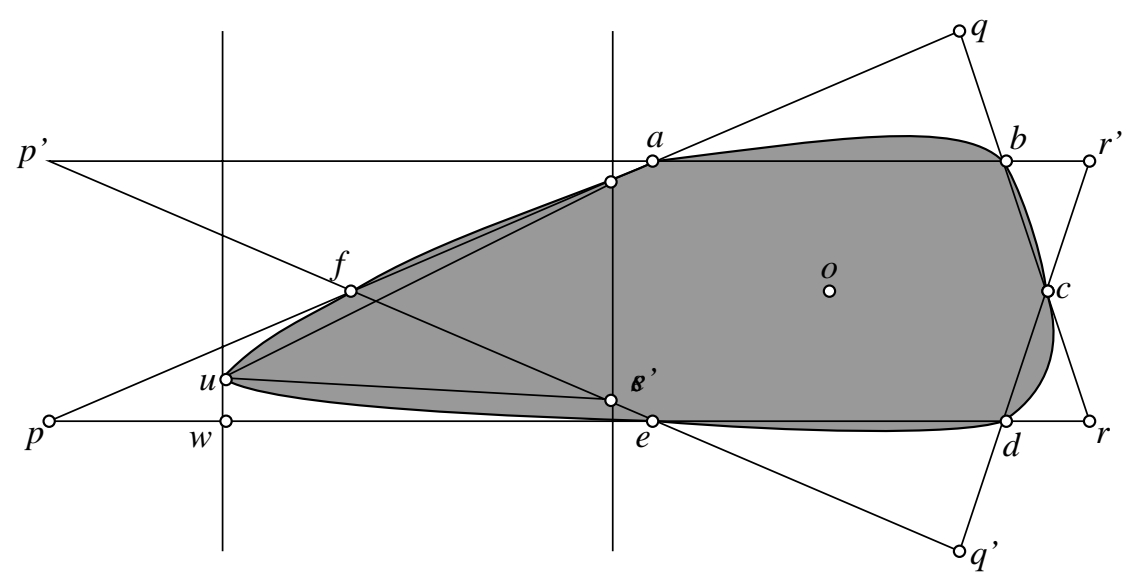

FIGURE 3.

Figure 2). Moreover, $|p d|=|p e|+|e d|$. Those inequalities and equalities lead to $|e d| \leq|p e| \leq 2|e d|$. This and $\frac{1}{2}|p e| \leq|w e| \leq|p e|$ imply that $|e d| \leq|w e| \leq 2|e d|$.

Let $\sigma=|w e| /|e d|$. By the above-obtained inequalities we have $\frac{1}{2} \leq \sigma \leq 2$. The required line $L$ is parallel to $M$ and its position depends on $\sigma$. Below we consider two cases in which we determine the position of $L$. By $C_{1}$ we denote the part of $C$ which is the intersection of $C$ with the halfplane bounded by $L$ and containing the point $u$. Let $C_{1}^{\prime}$ denote the image of $C_{1}$ after folding it along $L$.

Case 1 , when $1 \leq \sigma \leq 2$. We provide $L$ such that it bisects the segment $w d$ (see Figure 3). Denote by $l$ and $m$ the intersections of $L$ with the segments $a u$ and $e u$, respectively. Let $k$ and $n$ denote the intersections of $L$ with the boundary of $C$ (the notation is chosen such that $\mathrm{km}$ contains $l$ ). Through $k$ and $n$ we provide straight lines parallel to $a b$. Observe that the strip between them contains $C_{1}$ (if we assume that a point of $C_{1}$ is out of this strip, then the convexity of $C$ and $a, e \in C$ lead to the false conclusion that $k$ or $n$ is not in the boundary of $C$ ). Consequently, the strip also contains $C_{1}^{\prime}$. This and the choice of $L$ imply that $C_{1}^{\prime} \subset C$.

Denote by $v$ and $z$ the orthogonal projections of $u$ on the sgments ae and $l m$, respectively. We have $|u z|=\frac{1}{2}(|w e|+|e d|)=\frac{1}{2}(\sigma|e d|+|e d|)=\frac{1}{2}(1+\sigma)|e d|$. From the similarity of triangles $l m u$ and $a e u$, and from $|u v|=|w e|=\sigma|e d|$, we get $|l m| /|a e|=|u z| /|u v|=\left(\frac{1}{2}(1+\sigma)|e d|\right) /(\sigma|e d|)$. Thus $|l m|=\frac{1}{2}(1+\sigma) \sigma^{-1}|a e|$. Consequently, $|l m u|=\frac{1}{2}|l m| \cdot|u z|=\frac{1}{8}(1+\sigma)^{2} \sigma^{-1}|a e| \cdot|e d|$. From $|H|=\frac{3}{2}|a e| \cdot|e d|$ we obtain $|\operatorname{lmu}|=\frac{1}{12}(\sigma+1)^{2} \sigma^{-1}|H|$. Since $1 \leq \sigma \leq 2$, this is at least $\frac{1}{3}|H|$. Of course, $C_{1}$ contains the triangle $l m u$. From the inequality $|H| \geq \frac{2}{3}|C|$ established in Theorem 2 we obtain that $\left|C_{1}\right| \geq \frac{2}{9}|C|>\frac{1}{8}|C|$.

Case 2, when $\frac{1}{2} \leq \sigma \leq 1$. We provide the line $L$ through points $a$ and $e$. From the convexity of $C$ and since the axially regular hexagon abcdef is inscribed in $C$, we conclude that $C_{1}$ is in the strip between the straight lines containing the segments $a b$ and $c d$. This and $\sigma \leq 1$ imply $C_{1}^{\prime} \subset C$.

We provide supporting lines of $C$ through $b$ and $d$. They cut off four triangles from $S$. Since $|q b|=|b c|$ and since $|a b| \geq\left|b r^{\prime}\right|$, we see that the area of the two triangles cut off from $S$ by the supporting line through $b$ is minimized when the line contains the segment $b c . \quad|a b q|=\frac{1}{6}|H|$. Analogously, the area of the two 
triangles cut off by the second line is minimized when this line contains $c d$. Thus $|C| \leq\left|C_{1}\right|+\left|a q c q^{\prime} e\right|$. Since $\left|a q c q^{\prime} e\right|=|a b d e|+|a q b|+|b c d|+\left|e d q^{\prime}\right| \leq \frac{7}{4}|a b d e|$ and since $|H|=\frac{3}{2} \mid$ abde $\mid$, we get $|C| \leq\left|C_{1}\right|+\frac{7}{6}|H|$. Hence $|C| /\left|C_{1}\right| \leq 1+\frac{7}{6}\left(|H| /\left|C_{1}\right|\right)$. Moreover, we have $\left|C_{1}\right| \geq \mid$ aef $\left|\geq \frac{1}{6}\right| H \mid$. Consequently, $|C| /\left|C_{1}\right| \leq 8$. Thus $\left|C_{1}\right| \geq$ $\frac{1}{8}|C|$.

Theorem 3 leads to the following mirror image problem.

Problem. How large a portion (by volume) of any convex body $C \subset E^{d}$ may be folded into $C$ by a reflexion about a hyperplane?

The double volume of this largest portion of the volume of $C$ is a very natural measure of symmetry of $C$ with respect to a hyperplane (i.e. a measure of axiality for $d=2$ ). Recall that such measures are considered in [12], [3] and [5].

Another open problem is about a possibly small, with respect to the area, axially symmetric convex body containing a given convex body $C \subset E^{2}$. Of course, such an axially symmetric body of area at most $2|C|$ always exists; for instance we can take an ellipse (by the classic theorem of John [14]), a rectangle (see [17]), or a rhombus (see Corollary 1). We can get a slight improvement of the ratio 2 by lessening the rhombus $R^{\prime}$ up to the axially symmetric pentagon $P=R^{\prime} \cap T^{\prime}$ (here and below we use the notation of the proof of Corollary 2). Remember that $|C| \geq \rho|R|$. From the definition of $P$ and from $\frac{1}{2} \kappa^{2} \geq \frac{1}{8} \rho^{2}-\frac{1}{4} \rho+\frac{1}{8}$ we obtain that $|P| \leq\left(\frac{7}{8} \rho^{2}+\frac{1}{4} \rho-\frac{1}{8}\right)|R|$. Hence $|P| /|C| \leq \frac{7}{8} \rho+\frac{1}{4}-\frac{1}{8} \rho^{-1}$. Since $\rho \in[1,2]$, this value is always at most $\frac{31}{16}$. Thus we obtain the following corollary.

Corollary 3. Every convex body $C \subset E^{2}$ is contained in an axially symmetric convex body of area at most $\frac{31}{16}|C|$.

\section{REFERENCES}

[1] A. S. Besicovitch, Measure of a asymmetry of convex curves, J. London. Math. Soc. 23 (1948), 237-240. MR 10:320r

[2] A. B. Buda and K. Mislow, On measure of axiality for triangular domains, Elem. Math. 46 (1991), 65-73. MR 92d:52017

[3] G. D. Chakerian and S. K. Stein, Measures of symmetry of convex bodies, Canad. J. Math. 17 (1965), 497-504. MR 31:1612

[4] C. M. Christensen, Kvadrat indskrevet $i$ convex figur, Mat. Tidsskr. B (1950), 22-26. MR 12:525f

[5] B. A. deValcourt, Measures of axial symmetry for ovals, Bull. Amer. Math. Soc. 72 (1966), 289-290. MR 32:4596

[6] B. A. deValcourt, Measures of axial symmetry for ovals, Isr. J. Math. 4 (1966), 65-82. MR 34:3439

[7] B. A. deValcourt, Axially symmetric polygons inscribed in and circumscribed about convex sets, Elem. Math. 22 (1967), 121-133. MR 37:2093

[8] E. G. Eggleston, Convexity, Cambridge University Press, Cambridge, 1969. (1st ed. 1958, MR 23:A2123)

[9] A. Emch, Some properties of closed convex curves in a plane, Amer. J. Math. 35 (1913), 407-412.

[10] R. Fleischer, K. Mehlhorn, G. Rote, E. Welzl, and C. Yap, Simultaneous inner and outer approximation of shapes, Algorithmica 8, 1992, 365-389. MR 93j:68213

[11] W. Gross, Über affine Geometrie XIII: Eine Minimumeigenschaft der Ellipse und des Elipsoids, Ber. Ver. Sächs. Akad. Wiss. Leipzig. Math.-Nat. Kl. 70 (1918), 38-54.

[12] B. Grünbaum, Measures of symmetry of convex sets, Convexity (Seattle 1961), in Proc. Sympos. Pure Math. 7, Amer. Math. Soc., Providence, R.I., 1963, pp. 233-270. MR 27:6187

[13] J. L. Hodges, Jr., An extremal problem of geometry, J. London Math. Soc. 26 (1951), 312-313. MR 14:495f 
[14] F. John, Extremum problems with inequalities as subsidiary conditions, Courant Anniversary Volume 1948, pp. 187-204. MR 10:719b

[15] F. Krakowski, Bemerkung zur einer Arbeit von W. Nohl, Elem. Math. 18 (1963), 60-61.

[16] M. Lassak, Approximation of convex bodies by triangles, Proc. Amer. Math. Soc. 115 (1992), 207-210. MR 92h:52004

[17] M. Lassak, Approximation of convex bodies by rectangles, Geom. Dedicata 47 (1993), 111-117. MR 94f:52004

[18] W. Nohl, Die innere axiale Symmetrie zentrischer Eibereiche der euklidischen Ebene, Elem. Math. 17 (1962), 59-63.

[19] O. Schwarzkopf, U. Fuchs, G. Rote and E. Welzl, Approximation of convex figures by pairs of rectangles, Proc. 7-th Sympos. Teoret. Aspects Comput. Sci., In STAC 90, pp. 240-249, Lecture Notes in Comput. Sci. 415, Springer, Berlin. MR 91e:68148

[20] O. Schwarzkopf, U. Fuchs, G. Rote and E. Welzl, Approximation of convex figures by pairs of rectangles, Comp. Geom. Theor. Appl. 10 (1998), 77-87. MR 99a:52019

[21] K. Zindler, Über konvexe Gebilde II, Monatsh. Math. 31 (1921), 25-56.

Instytut Matematyki i Fizyki ATR, 85-796 Bydgoszcz, Poland

E-mail address: lassak@mail.atr.bydgoszcz.pl

Current address: Institut für Informatik, FU Berlin, D-14195, Berlin, Germany 